\title{
An acquired-pseudo Bernard Soulier syndrome occurring with autoimmune chronic active hepatitis and anti-cardiolipin antibody
}

\author{
Ian L.P. Beales
}

Ealing Hospital NHS Trust, Uxbridge Road, Southall, Middlesex UB1 3HW, UK

\begin{abstract}
Summary: A case of autoimmune chronic active hepatitis with unusual extrahepatic manifestations is described. The patient exhibited marked thrombocytopenia and platelet aggregation dysfunction and morphological changes suggesting an acquired Bernard Soulier-type syndrome. This has not previously been described in association with chronic active hepatitis. The patient also demonstrated significant titres of anti-cardiolipin antibodies. This is also a new finding in association with both the liver and platelet diseases. The platelet aggregation deficit was transferable in the patient's serum rather than being intrinsic to the platelets. The three previously reported cases of acquired Bernard Soulier are reviewed and possible mechanisms for the platelet dysfunction are discussed.
\end{abstract}

\section{Introduction}

Autoimmune chronic active hepatitis is an uncommon disease and, although the aetiology and pathogenesis are not yet defined, ${ }^{1}$ immune-related damage is important and widespread immunological abnormalities have been described. ${ }^{2} \mathrm{~A}$ variety of abnormalities of platelet morphology and function are documented in association with various forms of liver disease, some of these are due to deficits in immunological regulation. ${ }^{3-5}$

This case report describes the association of autoimmune chronic active hepatitis with both an acquired platelet defect resembling BernardSoulier syndrome and the presence of anticardiolipin antibody. Acquired platelet abnormalities comprising thrombocytopenia, abnormal morphology and impaired aggregation likened to a pseudo-Bernard-Soulier syndrome have been seen previously in only three cases each with a different association, and an autoimmune basis may have been involved. ${ }^{6-8}$ Anti-cardiolipin antibody has also been described in association with a variety of conditions often with an autoimmune basis or tissue destruction. ${ }^{9}$ Although associated thrombocytopenia is often recognized, the pseudoBernard-Soulier changes have not been described previously.

Correspondence: I.L.P. Beales, B.Sc., M.R.C.P., Department of Gastroenterology, Hammersmith Hospital, Du Cane Road, London W12 0NN, UK. Accepted: 9 August 1993

\section{Case report}

A 30 year old female of West Indian origin presented with a one month history of jaundiced sclerae, dark urine, malaise and anorexia. Her past medical history was unremarkable and there were no recognized risk factors for liver disease in her social, sexual or drug history. Examination revealed jaundice, $4 \mathrm{~cm}$ smooth hepatomegaly and no features of chronic liver disease. Initial investigations showed; haemoglobin $125 \mathrm{~g} / \mathrm{dl}$, white cell count $14.6 \times 10^{9} / 1$ (normal differential); platelets $52 \times 10^{9} / 1$; blood film showed large bizarrely shaped platelets with confirmed thrombocytopenia; activated thromboplastin time 49 seconds (control 45 seconds); prothrombin time 18 seconds (control 16 seconds); bleeding time 2 minutes; electrolytes and renal function were normal; bilirubin $201 \mu \mathrm{mol} / \mathrm{l}$; albumin $31 \mathrm{~g} / \mathrm{l}$; aspartate transaminase (AST) 1,374 IU/l; gamma glutamyl transferase (GGT) $103 \mathrm{IU} / \mathrm{l}$; $\mathrm{C}$ reactive protein $11 \mathrm{mg} / \mathrm{l}$; ESR $110 \mathrm{~mm} /$ hour; plasma viscosity 3.29 (normal 1.60-1.72).

Over the next 2 months her condition generally improved although she remained jaundiced. Laboratory investigations showed fluctuations of bilirubin 34-201 $\mu \mathrm{mol} / \mathrm{l}$; AST 114-2,040 IU/l; GGT 82-247 IU/1; unrelated to her clinical state. Further investigations revealed no evidence of pregnancy, Wilson's disease, haemochromatosis or alpha-1-anti-trypsin deficiency. There was no serological evidence of infection with hepatitis viruses $A, B$ and $C$, or with other recognized infectious causes of hepatitis. There was a polyclonal increase in immunoglobulins. Antibody to smooth muscle was consistently negative, anti- 
nuclear factor (ANF) was detected intermittently with a maximum titre of 1:320. Complement components were reduced-C3;35\%, C4; $14 \%, \mathrm{CH}$ $50 ; 11 \%$ (all of normal respectively). Anticardiolipin antibodies were detected by ELISA technique; ${ }^{10}$ IgG 54 units (normal <9); IgM 14 $(<8)$. Antibodies to liver-specific protein (antiLSP) were detected at a titre of greater than $1: 2,400$ and against asialoglycoprotein receptor (antiASGP-R) at a titre of $1: 1,200$. The direct antiglobulin (Coombs') test was positive with only IgG binding to erythrocytes. Antibodies to doublestranded DNA and extractable nuclear antigens were absent. Liver biopsy showed features of chronic active hepatitis with areas of established cirrhosis.

The thrombocytopenia showed wide fluctuations, on occasions normal counts and morphology were seen but most of the time giant platelets were present. A blood film 2 years previously was reviewed and found to be normal. Examination of the bone marrow showed plentiful but abnormal megakaryocytes with great variation in size and apparent failure of platelet separation. Large megakaryocytic inclusions were seen approximating in size to the giant platelets in peripheral blood. The other cell lineages were normal. Electron microscopy of platelets was normal.

Platelet aggregation with all standard agents (ristocetin, adrenaline, collagen, adenosine diphosphate) was impaired and not corrected by mixing 50:50 with normal plasma. The Russell viper test for lupus anticoagulant was negative. High titres of platelet-associated immunoglobulin were detected by immunofluorescence. ${ }^{11}$ Levels of factor VIII:C and von Willebrand factor were normal.

Treatment with prednisolone $20 \mathrm{mg}$ daily was commenced with symptomatic improvement. After 3 months therapy relevant results were: platelets $204 \times 10^{9} / 1$; AST $290 \mathrm{IU} / 1$; IgG $54.2 \mathrm{~g} / \mathrm{l}$; anticardiolipin antibody IgG positive at 24 units; anti-LSP > 1:2,400; anti-ASGP-R 1:1,300. Very occasional giant platelets were seen on the blood film.

The aggregation studies were repeated. Ristocetin aggregation was absent, collagen-induced aggregation reduced, other agonists normal. Aggregation with ristocetin was restored by suspending the patient's washed platelets in control plasma. The patient's plasma abolished ristocetin-induced aggregation of normal platelets.

\section{Discussion}

Autoimmune chronic active hepatitis (aCAH) is a grouping of non-homogeneous conditions with several similar features. Diagnosis is reached by the combination of: histology (showing piecemeal nec- rosis plus or minis cirrhosis); immunological abnormalities including the presence of circulating autoantibodies; the absence of other causes of chronic hepatitis; and the response to immunosuppression. Three sub-groups can be distinguished serologically by the pattern of serum antibodies directed against liver and kidney microsomal (LKM), liver cytosolic ( $\mathrm{LCl}$ ) and soluble liver antigens (SLA).$^{12}$ These are not routinely available as diagnostic tests but the overall diagnosis of autoimmune chronic active hepatitis is not in doubt. The presence of anti-LSP is associated with active disease and its detection is invariably associated with relapse. ${ }^{13}$ Anti-asialoglycoprotein receptor antibody is found in $49 \%$ of aCAH cases and the titre is related to disease activity. ${ }^{14}$

In addition to these specific antibodies a wide variety of other autoantibodies have been documented in aCAH; anti-thyroid; anti-parietal cell; anti-erythrocyte; anti-platelet. ${ }^{15,16}$ Anti-cardiolipin antibodies have not been previously associated with aCAH. An association of these latter antibodies has been found with many conditions not all having an autoimmune basis. In many cases the exact significance of the relationship is unclear. ${ }^{17}$ Despite the presence of the anticardiolipin antibody in significant titre and the thrombocytopenia there were no other features of the anti-phospholipid syndrome. ${ }^{18}$ There were insufficient features to make a diagnosis of systemic lupus erythematosus. ${ }^{19}$ The anti-cardiolipin antibody may have been generated as a nonspecific response to tissue injury, in this case hepatitis, as has been described following myocardial infarction rather than as an integral part of the autoimmune disease. ${ }^{20}$

Thrombocytopenia of varied degree is common in liver disease and is multifactorial in origin. ${ }^{21}$ In this case the thrombocytopenia, large abnormal platelets and impaired aggregation are suggestive of Bernard Soulier syndrome. ${ }^{22}$ The bleeding time was normal, a blood film 2 years previously was normal and there were no significant abnormalities on her parents' blood films, indicating she did not have true inherited Bernard Soulier or von Willebrand's disease. The aggregation defect and morphology are not normally found in autoimmune thrombocytopoenic purpura (ITP). ${ }^{23}$

There are three previous reports of an acquired pseudo-Bernard Soulier each with slightly different features and associations. One was found with juvenile myelodysplasia ${ }^{6}$ and one each with a lymphoproliferative disorder ${ }^{7}$ and procainamideinduced lupus. ${ }^{8}$ These acquired disorders showed impaired aggregation to ristocetin alone amongst the standard agonists and thrombocytopenia and the morphological changes were not consistent between the three cases. In contrast this case showed a wider scope of platelet function defect 
with impaired aggregation to all agonists. The exact changes in inherited and acquired Bernard Soulier remain unclear. ${ }^{22}$ The mechanism of thrombocytopenia in this case may be multifactorial, despite the absence of splenomegaly splenic pooling could contribute. ${ }^{21}$ Platelet-associated immunoglobulins (PA-Ig) are common in aCAH and thrombocytopenia is rare in the absence of PA-Ig. ${ }^{15}$ In contrast to autoimmune thrombocytopenic purpura there is no correlation between antibody titre and platelet count. ${ }^{15}$ In addition to antibody directed against platelet antigens some PA-Ig is found as immune complexes binding non-specifically to platelet $\mathrm{Fc}$ receptors. Both types of PA-Ig are associated with thrombocytopenia and are difficult to differentiate by standard immunofluorescence techniques..$^{24,25}$ In the presence of severe hypergammaglobulinaemia and complement consumption, it is possible that immune complex binding (with target antigen unclear) with subsequent complement activation and specific anti-platelet antibodies are both contributing to platelet destruction.

The platelet defect in Bernard Soulier syndrome involves a deficiency in glycoprotein Ib/IX preventing interaction in Von Willebrand factor dependent adhesion to the sub-endothelium. ${ }^{26}$ The aggregation studies in this patient show that the abnormality is transmissible in the serum rather than the platelets themselves. It seems likely an autoantibody is responsible in this case. Antibody directed against $\mathrm{gp} \mathrm{Ib} / \mathrm{IX}$ has been documented in immune thrombocytopenia without the described morphological change or aggregation deficit. ${ }^{27}$ Of the three cases of pseudo-Bernard Soulier, two demonstrated transmission of the impaired ristocetin response in the immunoglobulin fraction ${ }^{7,8}$ (in the other the defect was in the platelet). Both of these cases showed PA-Ig. One of these antibodies was directed against an epitope which did not seem

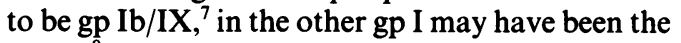
target. $^{8}$

\section{References}

1. Sharon, N. \& Eddleston, A. Autoimmune chronic active hepatitis. In: McIntyre, N., Benhamou, J.-P., Bircher, J. Rizzettol, M. \& Rodes, J. (eds) Oxford Textbook of Clinical Hepatology. Oxford University Press, Oxford, 1991, pp. 758-767.

2. Macfarlane, I. \& Williams, R. Liver membrane antibodies. $J$ Hepatol 1985, 1: 313-319.

3. Thomas, D., Ream, V. \& Stuart, R. Platelet aggregation in patients with Laennec's cirrhosis of the liver. $N$ Engl J Med 1967, 276: 1344-1348.

4. Barrison, I., Knight, I., Viola, L., Boots, M., Murray-Lyon, I. \& Mitchell, T. Platelet associated immunoglobulin in chronic liver disease. Br J Haem 1981, 48: 347-350.

5. Rubin, M. Platelet function in chronic liver disease; relationship to disease activity. Dig Dis Sci 1979, 24: 197-202.
Given the widespread immunological abnormalities in this case it seems likely that PA-Ig was involved in the aggregation defect. The impaired response was to a range of agonists rather than just ristocetin. It is possible that there are different antibodies directed against different platelet membrane antigens involved in the aggregation responses. It has not yet been possible to characterize the target antigens. It has been suggested that in ITP there may be more than one platelet target site per patient. ${ }^{28}$ Previously antibody-associated platelet dysfunction without the pseudo-Bernard Soulier changes have been described, although the targets antigens were not identified. ${ }^{29}$ The bone marrow appearances may reflect anti-platelet/ megakaryocyte antibody action although there is no direct evidence of this.

The immunopathogenesis of aCAH is not specifically defined. Widespread immunological abnormalities in extrahepatic systems are known. This is the first case associated with anti-cardiolipin antibody, although the significance of this in relation to the disease and thrombocytopenia is unclear. Platelet morphological and functional deficits described in this case fit with the few previous cases of pseudo-Bernard Soulier syndrome and appear to be antibody mediated. The target antibodies have not been fully defined. This is the first case of acquired Bernard Soulier associated with autoimmune chronic active hepatitis and should lead to greater consideration of anti-platelet antibodies in aCAH.

\section{Acknowledgements}

I would like to thank Dr U. Hegde for reviewing the haematology and advice, Dr I. Lampert for reviewing the histology, Dr M. Rudolf for allowing me to report this patient and King's College Hospital, London, for performing the assays for the anti-LSP and anti-ASGP-R antibodies.

6. Bernt, M., Kabal, A., Grimsby, P. et al. An acquired Bernard-Soulier like platelet defect associated with juvenile myelodysplastic syndrome. $\mathrm{Br} J$ Haem 1988, 68: 97-101.

7. Devine, D., Currie, M., Rosse, W. \& Greenburg, C. PseudoBernard-Soulier caused by antibody to glycoprotein $\mathrm{gp} \mathrm{lb}$. Blood 1987, 70: 428-430.

8. Sticker, R., Wong, D., Saks, S. et al. Acquired Bernard-Soulier syndrome. Evidence for a role of a 210,000 molecular weight protein in interaction of platelets and Von Willebrand factor. J Clin Invest 1985, 76: 1274-1278.

9. Cervera, R., Font, J., Khamnashta, A. \& Hughes, G.R.V. Antiphospholipid antibodies: which and when? Postgrad Med J 1990, 66: 889-891. 
10. Loizou, S., McCree, J., Rudge, J. et al. Measurement of anticardiolipin antibodies by an immunosorbent assay (ELISA). Standardisation and quantitation of results. Clin Exp Immunol 1985, 62: 738-745.

11. Von De Born, A., Vos, J., Van Der Lelie, J., Bossers, B. \& Van Dalen, C. Clinical significance of positive platelet immunofluoresence test in thrombocytopenia. $\mathrm{Br} \mathrm{J} \mathrm{Haem}$ 1986, 64: 767-776.

12. Mitchison, H. \& Bassendine, M. Autoimmune liver disease. In: Pounder, R.E. (ed) Recent Advances in Gastroenterology 8. Churchill Livingstone, London, 1992, pp. 225-239.

13. Macfarlane, I., McSurley, C., Hegarty, J. et al. Antibodies to liver specific protein predict outcome of treatment withdrawal in autoimmune chronic active hepatitis. Lancet 1984, ii: 954-956.

14. Treichel, V., Lohr, H., Puralla, T. et al. Occurrence of antibodies and proliferating clones to human asialoglycoprotein receptor in autoimmune chronic active hepatitis. $J$ Hepatol 1989, 9: s90.

15. Landolfi, R., Leone, G., Feldeli, G., Storti, S., Laghi, F. \& Bizzi, B. Platelet associated IgG in acute and chronic hepatic diseases. Scand J Haematol 1980, 25: 417-422.

16. Doniach, D., Roitt, I., Walker, J. \& Sherlock, S. Tissue antibodies in primary biliary cirrhosis, active chronic hepatitis, cryptogenic cirrhosis and other liver diseases. Clin Exp Immunol 1966, 1: 237-262.

17. Asherson, R.A. \& Harris, E.N. Anticardiolipin antibodies-Clinical associations. Postgrad Med J 1986, 62: 1081-1087.

18. Hughes, G., Harris, E. \& Gharawi, A. Anticardiolipin syndrome. $J$ Rheum 1986, 13: 486-488.

19. Tan, E., Cohen, A., Fries, J. et al. 1982 revised criteria for the classification of systemic lupus erythematosus. Arthritis Rheum 1982, 25: 1271-1277.

20. Hamsten, A., Norberg, R., Bjorkholm, M., De Faire, U. \& Holm, G. Antibodies to cardiolipin in young survivors of myocardial infarction. An association with recurrent cardiovascular events. Lancet 1986, i: 113-162.
21. Schnidt, K., Rassmusen, J., Bekker, C. \& Madsen, P. Kinetics and in vivo distribution of 111-In-labelled autologous platelets in chronic hepatic disease; mechanisms of thrombocytopenia. Scand J Haematol 1985, 34: 39-49.

22. Bernt, M., Gregory, B., Chong, H., Zola, H. \& Castaldi, P Additional glycoprotein defects in Bernard Soulier's syndrome: confirmation of genetic basis by parental analysis. Blood 1983, 62: 800-807.

23. Bossel, J. \& Schreiber, A. Immune thrombocyopenic purpura. In: Hoffman, R., Benz, E., Shattil, S., Furie, B. \& Cohen, H. (eds) Hematology, Basic Principles and Practice. Churchill Livingstone, New York, 1991, pp. 1485-1490.

24. Hegde, U. Platelet antibodies in immunethrombocytopoenia. Blood Rev 1992, 6: 34-42.

25. Trent, R., Clancy, R., Danis, V. \& Basten, A. et al. Immune complexes in thrombocytopenia. $\mathrm{Br} J$ Haem 1980, 44: 645-654.

26. Clemetson, K., McGregor, J. \& James, E. Characterisation of platelet membrane abnormalities in Bernard Soulier syndrome and comparison with normal by surface labelling techniques and high resolution 2-D gel electrophoresis. J Clin Invest 1983, 70: 304-311.

27. Szathowski, N., Kunicki, T. \& Aster, R. Identification of glycoprotein Ib as a target of autoantibody in idiopathic thrombocytopenic pupura. Blood 1986, 67: 310-315.

28. Nujent, D. Identification of antiplatelet antibody idiotypes associated with glycoprotein Ib specificity present in ITP plasma and produced from human hybridomas from ITP spleen cell fusions. Thromb Haemostasis 1987, 589: 531.

29. Moore, A., Ross, G. \& Nachman, R. Interaction of platelet membrane receptors with Von Willebrand factor, ristocetin and the Fc region of immunoglobulin G. J Clin Invest 1978, 62: $1053-1060$. 